whereas expression of TIMP1 and 3 was not altered. Next, we investigated which cell-type in the synovium may cause the increased MMP expression. Stimulation of synovial OA fibroblasts with Wnt3a increased the expression of MMP3 and MMP13, whereas WISP1 led to increased MMP3 expression. Stimulation of THP-1 cells with Wnt3a resulted in highly increased MMP3, MMP9 and MMP13 expression. WISP1 stimulation led to increased expression of MMP3. TIMP1 and 3 expression was not altered. Synovial overexpression of Wnt8a, Wnt16 and WISP1 in murine knee joints with use of adenoviral vectors led to cartilage damage in vivo. 7 days after overexpression, we found a significant induction of $O A$ pathology at the medial margin of the medial tibial plateau, a preferential site for damage in experimental OA. Lesions were found in $92 \%$ $(\mathrm{n}=12)$ of the knee joints after Wnt8a overexpression compared to $17 \%(\mathrm{~N}=12)$ for the control virus and $80 \%(\mathrm{~N}=5)$ for $\mathrm{Wnt} 16$ overexpression, but only $20 \%(\mathrm{~N}=5)$ for the control virus. In addition, overexpression of WISP1 led to significantly increased cartilage damage after 7 days.

Conclusions Canonical Wnts produced in the synovium may play an important role in OA pathology by increasing the expression of cartilage-degrading MMPs in synovial tissue, where fibroblasts and macrophages showed comparable patterns of MMP induction. Synovium-specific overexpression of Wnt signalling members induces cartilage damage.

\section{A10.27 SYSTEMIC SCLEROSIS - ROLE OF AGONISTIC AUTOANTIBODIES DIRECTED AGAINST THE ANGIOTENSIN RECEPTOR TYPE 1 AND THE ENDOTHELIN RECEPTOR TYPE A ON IMMUNE CELLS IN DISEASE PATHOGENESIS}

doi:10.1136/annrheumdis-2013-203224.27

1,2J Günther, ${ }^{1,2} \mathrm{~A}$ Kill, ${ }^{2} \mathrm{MO}$ Becker, ${ }^{1,2} \mathrm{G}$ Riemekasten. ${ }^{1}$ German Rheumatism Research Centre, a Leibniz institute; ${ }^{2}$ Charité University Hospital, Berlin, Germany

Background and Objectives Agonistic autoantibodies against the angiotensin receptor type 1 (AT1R) and the endothelin receptor type A (ETAR) have been identified in the majority of systemic sclerosis (SSc) patients. We proved the expression of the AT1R and the ETAR on human peripheral immune cells. Here, we investigated the effects of these autoantibodies on human immune cells in vitro, the association with clinical data, and their possible role in the pathogenesis of systemic sclerosis.

Methods Peripheral blood mononuclear cells (PBMCs) from healthy donors were isolated by gradient centrifugation and in vitro stimulated by affinity-purified IgG from SSc patients containing anti-AT1R and anti-ETAR antibodies as well as by affinity-purified $\operatorname{IgG}$ from healthy donors. After stimulation the protein expression of cytokines was measured by ELISA. T cells were isolated from PBMCs by magnetically activated cell sort (MACS) and cultivated in antiCD3/CD28 coated plates. Afterwards, migration assays were performed towards IgGs of SSc patients or healthy donors.

Results IgG of SSc patients induced a significantly increased expression and secretion of IL-8 and an abundant but due to a broad range not significant secretion of CCL18. T cell migration towards IgG of SSc patients was increased. All these effects were significantly reduced by commercial AT1R and ETAR antagonist, confirming the specifity of the biological activity of the autoantibodies. Correlation analysis with clinical data of the SSc IgG donors revealed a negative correlation of IL-8 expression with the time since disease onset, and an association of CCL18 expression with the incidence of vascular complications. The chemotactic motility of $\mathrm{T}$ cells correlated with the autoantibody titers of the used SSc IgG.

Conclusions SSc patient IgGs containing anti-AT1R and antiETAR antibodies seem to have effects on inflammation and fibrosis. IL-8 is a major inflammatory cytokine, inducing migration and inflammatory effects. CCL18 is a fibrotic cytokine, also contributing to leucocyte infiltration, and to lung fibrosis in SSc and other diseases. Thus, anti-AT1R and anti-ETAR antibodies may play an important role particularly in the early stage of SSc, contributing to leucocyte infiltration, vascular inflammation and injury, which are regarded to be the first events leading to tissue damage and fibrosis. 